\title{
The influence of type 1 diabetes on pancreatic weight
}

\author{
Martha L. Campbell-Thompson ${ }^{1} \cdot$ John S. Kaddis $^{2} \cdot$ Clive Wasserfall $^{1}$ • \\ Michael J. Haller ${ }^{3}$ - Alberto Pugliese ${ }^{4}$. Desmond A. Schatz ${ }^{3}$. \\ Jonathan J. Shuster ${ }^{5}$. Mark A. Atkinson ${ }^{1}$
}

Received: 28 May 2015 / Accepted: 18 August 2015 /Published online: 10 September 2015

(C) Springer-Verlag Berlin Heidelberg 2015

\begin{abstract}
Aims/hypothesis Previous studies of pancreases obtained at autopsy or by radiography note reduced pancreas weight (PW) and size, respectively, in type 1 diabetes; this finding is widely considered to be the result of chronic insulinopenia. This literature is, however, limited with respect to the influence of age, sex, anthropometric factors and disease duration on these observations. Moreover, data are sparse for young children, a group of particular interest for type 1 diabetes. We hypothesised that the pancreas-to-body weight ratio would normalise confounding inter-subject factors, thereby permitting better characterisation of PW in type 1 diabetes.

Methods Transplant-grade pancreases were recovered from 216 organ donors with type 1 diabetes $(n=90)$, type 2 diabetes $(n=40)$ and no diabetes $(n=86)$. Whole-organ and
\end{abstract}

Electronic supplementary material The online version of this article (doi:10.1007/s00125-015-3752-z) contains peer-reviewed but unedited supplementary material, which is available to authorised users.

Mark A. Atkinson

atkinson@ufl.edu

1 Department of Pathology, Immunology, and Laboratory Medicine, College of Medicine, University of Florida, POB 100275, 1395

Center Drive, Gainesville, FL 32610, USA

2 Department of Information Sciences, City of Hope, Duarte, CA, USA

3 Department of Pediatrics, College of Medicine, University of Florida, Gainesville, FL, USA

4 Diabetes Research Institute, Miller School of Medicine, University of Miami, Miami, FL, USA

5 Department of Health Outcomes and Policy, College of Medicine, University of Florida, Gainesville, FL, USA head, body and tail weights were determined. The relative PW (RPW; PW [g] / body weight [kg]) was calculated and tested for normalisation of potential differences due to age, sex and BMI.

Results PW significantly correlated with body weight in control donors $\left(R^{2}=0.76, p<0.001\right)$ while RPW $(1.03 \pm 0.36$, mean $\pm \mathrm{SD})$ did not significantly differ across ages ( $0-58$ years). Donors with type 1 diabetes $(0.57 \pm 0.18$, $p<0.001)$, but not those with type 2 diabetes $(0.93 \pm 0.30)$, had significantly lower RPW. The relative weights of each pancreatic region from donors with type 1 diabetes were significantly smaller than those of regions from control donors and donors with type 2 diabetes $(p<0.001)$. Perhaps most interestingly, the RPW was not significantly associated with duration of type 1 diabetes or type 2 diabetes.

Conclusions/interpretation RPW allows for comparisons across a wide range of donor ages by eliminating confounding variables. These data validate an interesting feature of the type 1 diabetes pancreas and underscore the need for additional studies to identify the mechanistic basis for this finding, including those beyond the chronic loss of endogenous insulin secretion.

Keywords Children · Organ donor · Pancreas · Pancreas weight $\cdot$ Type 1 diabetes $\cdot$ Type 2 diabetes
Abbreviations
BSA Body surface area
nPOD Network for Pancreatic Organ donors with Diabetes
PW Pancreas weight
RPW Relative PW
UNOS United Network for Organ Sharing 


\section{Introduction}

Type 1 diabetes is an autoimmune disorder resulting in the destruction of insulin-producing pancreatic beta cells [1]. Reduced pancreas weights (PWs) at autopsy have been demonstrated in patients with recent-onset type 1 diabetes as well as in those with long-standing disease (reviewed in [1, 2]). Non-invasive imaging has also demonstrated reduced pancreas volume in patients with recent-onset diabetes [3, 4]. The mechanisms underlying reduction in pancreatic size have largely been hypothesised to involve loss of insulinotrophic effects on the exocrine pancreas as islets constitute only $1-2 \%$ of the entire pancreas volume [5,6]. The purpose of this study was to examine the influence of a series of physiological and anthropometric factors, as well as disease duration, on PW in high-quality (i.e. transplant-grade, not autopsy) pancreases obtained from a large number of organ donors with type 1 diabetes, type 2 diabetes and no diabetes. Furthermore, relative PW (RPW; PW [g] / body weight [kg]) was calculated for each pancreatic region (head, body and tail) and the entire organ with subsequent analysis for correlations to age, sex, BMI and diabetes duration.

\section{Methods}

Donors The JDRF Network for Pancreatic Organ donors with Diabetes (nPOD) programme recovered pancreases from organ donors as previously described [7]. Donors without diabetes (controls, $n=86$ ) had no medical history of pancreatic disease and included neonates and children. Donors with type 1 diabetes $(n=90)$ or type 2 diabetes $(n=40)$ were classified according to ADA guidelines following medical chart review in concert with islet autoantibody and C-peptide assays, as previously described [7]. Demographic data (age, sex, ethnicity, body weight, BMI) and other clinical findings were recorded from terminal hospital records or data provided by the United Network for Organ Sharing (UNOS) and are summarised in Table 1. Four donors from the Harvard Joslin Medalist programme had pancreases recovered at autopsy and were processed by nPOD [8]. All procedures were conducted in compliance with UNOS and the University of Florida Institutional Review Board requirements, based upon federal guidelines.

Pancreas processing Pancreases were recovered as if for transplantation, shipped by organ courier in iced transport media and cleaned of all extraneous tissues by a licensed pathology assistant according to standard procedures [7]. The pancreas was weighed intact and/or after dividing it into three regions (head, body and tail) by cutting the junction between head and body at the notch and dividing the remaining portion in half for body and tail regions.
Statistical analysis The primary analysis was based upon RPW as we expected this to minimise the confounding influences of age, sex and BMI on PW, particularly for individuals of younger age with high incidence of type 1 diabetes. BMI $\left(\mathrm{kg} / \mathrm{m}^{2}\right)$ was calculated as weight / (height squared). Body surface area $\left(\mathrm{BSA} ; \mathrm{m}^{2}\right)$ was calculated as $0.0235 \times$ height $(\mathrm{cm})^{0.42} \times$ weight $(\mathrm{kg})^{0.51}$. Similarly, secondary analysis contrasted relative regional weights in the same manner. To control study-wide errors in the primary analysis, the three groups were first compared by ANOVA followed by a Bonferroni corrected contrast between all pairs. Relative regional weights were analysed in the same manner. As a tertiary contrast, we compared the proportions of the total PWs for the three regions across the three donor groups. For analyses not involving RPW, all $p$ values were unadjusted, two-tailed and statistically significant if $<0.05$. A $p$ value was not calculated if values from two or more cells were equal to 0. Statistical analysis was performed using SAS software version 9.3 (SAS Institute, Cary, NC, USA) and GraphPad Prism (GraphPad Software, San Diego, CA, USA).

\section{Results}

Group demographic and clinical variables A total of 216 donors ( $n=86$ control, $n=90$ type 1 diabetes, $n=40$ type 2 diabetes) were prospectively recovered during this study (Table 1). As expected, the ages, heights, weights and BMIs of the donors were significantly different between groups due to the inclusion of very young children in the control group (Table 1). Donors with type 1 diabetes had disease durations of $0-84$ years while donors with type 2 diabetes had disease durations ranging from 0 to over 50 years (Table 1). Among those with type 1 diabetes, 15 tested negative for autoantibodies, 36 positive for one and 36 positive for two or more. In those with type 2 diabetes, ten had antibodies against insulin, consistent with medical history of insulin injections and likely reflecting responses to parenteral insulin rather than islet autoantibodies. One donor with type 2 diabetes had low-titre GAD autoantibodies, yet all other findings were consistent with a type 2 diabetes diagnosis (e.g. C-peptide level of $11.55 \mathrm{ng} / \mathrm{ml}$, history of metformin treatment, islet amyloidosis by histopathology [case 6149]).

PW vs RPW In controls, raw PW correlated closely with age in young donors until adulthood (second order polynomial, $R^{2}=0.82, n=86$; Fig. 1a). PW was significantly correlated with body weight (slope $=1.8, R^{2}=0.56, p<0.001$; Fig. 1b) and normalisation of PW to body weight removed any association with age $\left(R^{2}=0.07\right.$; Fig. 1a). Raw PWs showed significant differences between all three groups $(50.6 \pm 35.9$ controls, $70.3 \pm 17.8$ type 1 diabetes, $96.4 \pm 16.8$ type 2 diabetes). 
Table 1 Donor characteristics

\begin{tabular}{|c|c|c|c|c|c|c|c|}
\hline \multirow[t]{2}{*}{ Characteristic } & \multicolumn{2}{|c|}{ Controls } & \multicolumn{2}{|c|}{ Type 1 diabetes } & \multicolumn{2}{|c|}{ Type 2 diabetes } & \multirow[t]{2}{*}{ Overall $p$ value } \\
\hline & $n$ & Value & $n$ & Value & $n$ & Value & \\
\hline Age, years & 86 & $16.3(14.8)$ & 90 & $32.2(16.2)$ & 40 & $48.9(14.9)$ & $<0.001$ \\
\hline Sex & 86 & & 90 & & 40 & & 0.21 \\
\hline Female & 28 & 33 & 38 & 42 & 19 & 47 & \\
\hline Male & 58 & 67 & 52 & 58 & 21 & 53 & \\
\hline Ethnicity & 86 & & 90 & & 40 & & $<0.01$ \\
\hline Black/African-American & 15 & 17 & 9 & 10 & 11 & 27 & \\
\hline White & 59 & 69 & 74 & 82 & 18 & 45 & \\
\hline Hispanic/Latino & 11 & 13 & 7 & 8 & 9 & 23 & \\
\hline Other $^{\mathrm{a}}$ & 1 & 1 & 0 & 0 & 2 & 5 & \\
\hline Height, cm & 86 & $132.8(49.8)$ & 90 & $167.7(16.7)$ & 40 & $170.8(10.5)$ & $<0.001$ \\
\hline Body weight, kg & 86 & $50.6(35.9)$ & 90 & $70.5(17.8)$ & 40 & $96.4(16.8)$ & $<0.001$ \\
\hline BMI & 86 & $22.5(6.9)$ & 90 & $24.7(4.3)$ & 40 & $33.0(6.1)$ & $<0.001$ \\
\hline Autoantibody results ${ }^{\mathrm{b}}$ & 77 & & 87 & & 39 & & $<0.001^{\mathrm{c}}$ \\
\hline Negative & 77 & 100 & 15 & 17 & 28 & 76 & \\
\hline \multicolumn{8}{|l|}{ Single positive } \\
\hline IAA & 0 & 0 & 29 & 36 & 10 & 21 & \\
\hline GADA & 0 & 0 & 4 & 1 & 1 & 3 & \\
\hline $\mathrm{IA} 2 \mathrm{~A}$ & 0 & 0 & 1 & 1 & 0 & 0 & \\
\hline ZnT8A & 0 & 0 & 2 & 2 & 0 & 0 & \\
\hline \multicolumn{8}{|l|}{ Two or more positives } \\
\hline Including IAA & 0 & 0 & 34 & 41 & 0 & 0 & \\
\hline Excluding IAA & 0 & 0 & 2 & 2 & 0 & 0 & \\
\hline C-peptide ${ }^{\mathrm{d}}, \mathrm{ng} / \mathrm{ml}$ & 77 & $5.6(4.9)$ & 85 & $0.2(0.6)$ & 39 & $4.6(5.9)$ & $<0.001$ \\
\hline Diabetes duration, years & NA & NA & 89 & $18.6(14.9)$ & 34 & $11.1(10.1)$ & NR \\
\hline Hospital duration $^{\mathrm{e}}$, days & 80 & $5.0(3.7)$ & 80 & $4.3(2.7)$ & 37 & $4.8(3.9)$ & 0.38 \\
\hline Organ transit time ${ }^{\mathrm{f}}, \mathrm{h}$ & 84 & $16.2(6.8)$ & 87 & $17.1(5.6)$ & 39 & $16.1(4.9)$ & 0.54 \\
\hline Cause of death, $\%$ & 86 & & 89 & & 40 & & NR \\
\hline Anoxia & 30 & 35 & 39 & 44 & 11 & 28 & \\
\hline Cerebrovascular/stroke & 14 & 16 & 22 & 25 & 25 & 62 & \\
\hline Head trauma & 36 & 42 & 19 & 21 & 2 & 5 & \\
\hline Other & 6 & 7 & 9 & 10 & 2 & 5 & \\
\hline
\end{tabular}

Values are presented as mean (SD) or as \% for categorical variables

${ }^{a}$ Includes two Asian donors with type 2 diabetes and one American Indian/Alaska Native control donor

${ }^{\mathrm{b}}$ All values from RIA testing were converted to NIDDK units and defined as positive if one or more of the following applied: IAA $\geq 0.01$; GADA $\geq 20$; $\mathrm{IA} 2 \mathrm{~A} \geq 5$ or $\mathrm{ZnT} 8 \mathrm{~A} \geq 0.02$

${ }^{\mathrm{c}}$ Excludes group of donors with more than two autoantibodies

${ }^{\mathrm{d}}$ When C-peptide levels fell below the lower limit of detection (i.e. $<0.05$ ), a fill value of 0.001 was used

${ }^{\mathrm{e}}$ Period from admission time to intraoperative aortic cross clamp

${ }^{\mathrm{f}}$ Period from aortic cross clamp to laboratory receipt

The following were used to generate $p$ values: ANOVA (more than two groups compared) or Satterthwaite corrected $t$ test (two groups compared) if continuous; Pearsons's $\chi^{2}$ if nominal, categorical and values in all cells $>5$ or Freeman-Halton extension of Fisher's exact test if nominal, categorical and values in one or more cells $<5$

GADA, GAD autoantibody; IAA, insulin autoantibody; IA2A, islet antigen 2 autoantibody; NR, not reported; ZnT8A, zinc transporter 8 autoantibody

The RPW was examined to reduce inter-subject factors related to age, sex, BMI and duration of diabetes. None of 11 potential confounding variables were significant for
RPW. As such, use of RPW normalised PWs from very young donors to older donors and accounted for differences in sex and BMI. 
Fig. 1 RPW and diabetes. (a) In control donors without diabetes, PW (g) (triangles) correlated with age while RPW (squares) had no association with age. (b) PW (g) was significantly correlated with body weight $(\mathrm{kg})$ in controls. (c) Donors with type 1 diabetes (T1D, circles) had significantly lower RPW than controls (ND; triangles) and donors with type 2 diabetes (T2D, squares). (d) RPWs by pancreatic region shown for controls (ND; triangles) and donors with T1D (circles) or T2D (squares). Donors with T1D had significantly lower relative regional weights compared with controls and donors with T2D. Donors with T2D had significantly different relative tail weights compared with controls and donors with T1D. (e) RPW did not correlate with disease duration for donors with T1D (circles) or T2D (squares). Data are means $\pm \mathrm{SD} ; *<<0.05$ (T1D head vs ND and T2D head; T1D body vs ND and T2D body; T1D tail vs ND and T2D tail; T2D tail vs ND and T1D tail) and $* * * p<0.001$ (T1D vs ND and $\mathrm{T} 2 \mathrm{D}$ donors)
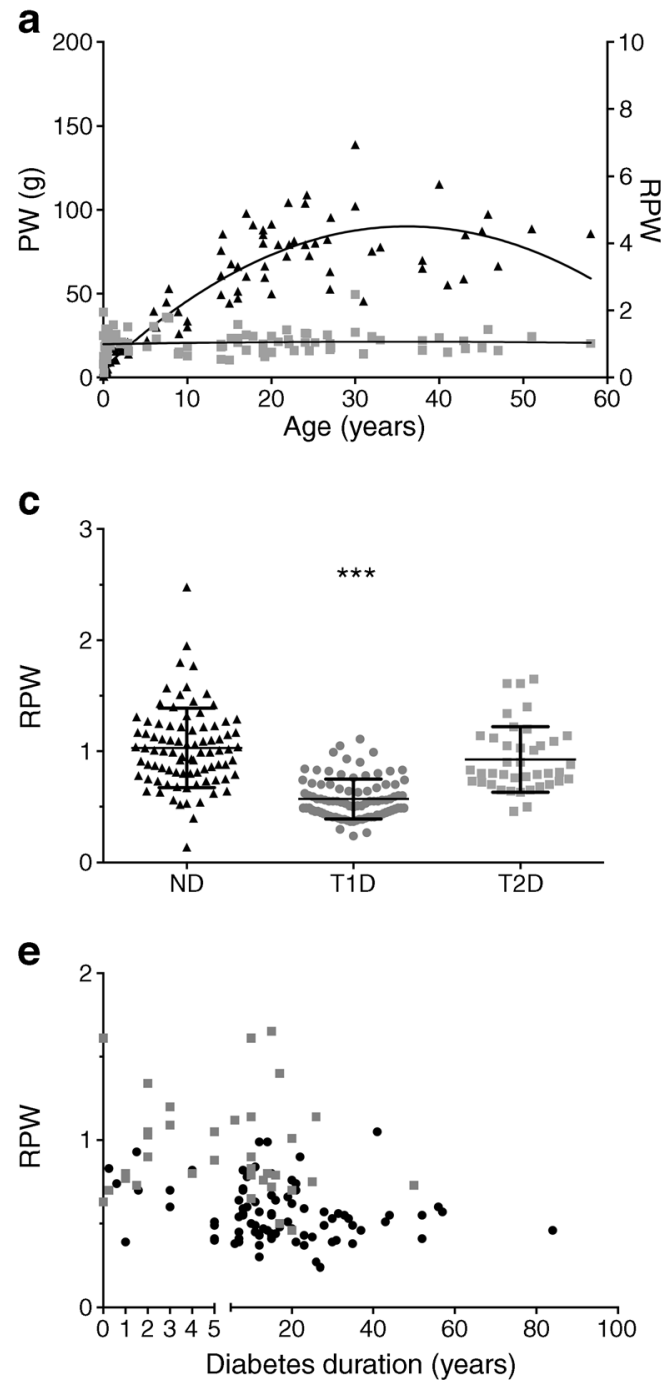
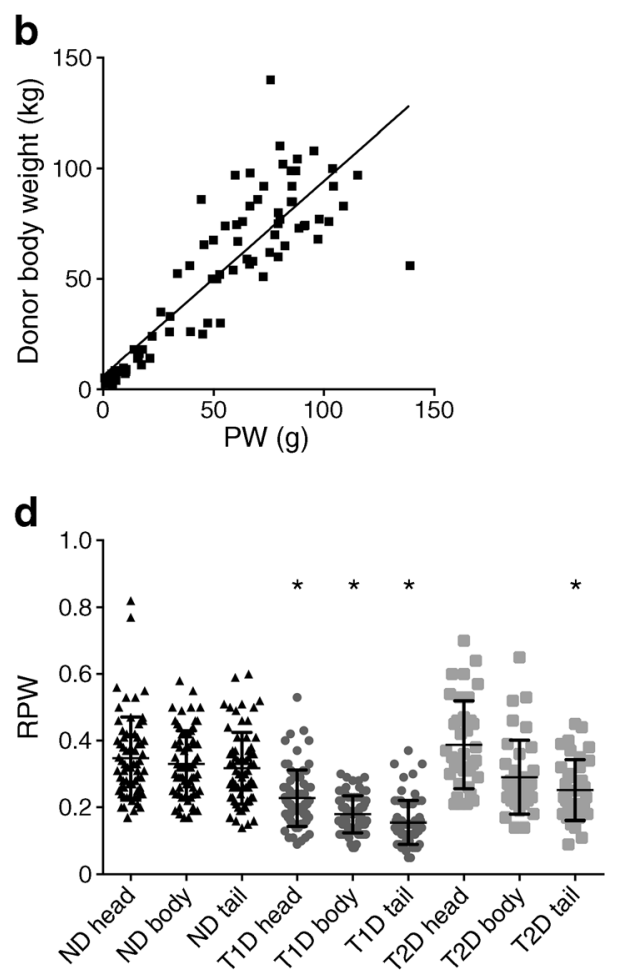

\section{Reduced RPW and relative regional weights in donors} with type 1 diabetes The RPW for donors with type 1 diabetes was $0.57 \pm 0.18(n=90)$ compared with $1.03 \pm 0.36$ for donors without diabetes $(n=86)$ and $0.93 \pm 0.30(n=40)$ for donors with type 2 diabetes ( $p<0.001$; Fig. $1 \mathrm{c})$. BSA $\left(\mathrm{m}^{2}\right)$ was also tested for PW normalisation (PW/BSA); similar results were obtained except that donors with type 2 diabetes had significantly higher PW/BSA compared with controls (electronic supplementary material [ESM] Fig. 1 a, c, e). The BSA was also converted from area to volume $\left(\mathrm{BSA}^{1.5}\right)$ and used to normalise PWs (PW/BSA ${ }^{1.5}$ ); similar proportions were found as for RPW (ESM Fig. 1 b, d, f). Correlations between RPW and PW/BSA ${ }^{1.5}$ were $\geq 0.94$ for all three groups.

Relative weights of each pancreatic region for donors with type 1 diabetes were significantly smaller than the respective regions of controls and donors with type 2 diabetes (Fig. 1d). The relative tail weights were also significantly different between all groups (Fig. 1d).
Diabetes duration and C-peptide levels do not correlate with RPW RPW was not significantly associated with duration of diabetes for donors with type 1 diabetes or type 2 diabetes, even for those with diabetes of short duration (Fig. 1e). RPW did not significantly correlate with C-peptide levels or diabetes duration in donors with type 1 diabetes who had detectable levels (ESM Fig. 2 a-f). Interestingly, raw PWs in controls were significantly though weakly correlated with C-peptide levels ( $r=0.33, p<0.01$, ESM Fig. 2e).

\section{Discussion}

Herein, we note that RPW allows for examination of PW differences between study subject groups despite differences in age, ethnicity or body weight. In donors with type 1 diabetes, RPW was only $55 \%$ that of donors without diabetes. Strikingly, this loss in weight was apparent at or near disease 
onset and was minimally altered in those with disease duration of several decades. This is an extremely interesting finding in that it suggests a different concept from the widely held notion that the reduced PW is a result of long-term insulin absence.

Our observation of pronounced PW loss in recent-onset type 1 diabetes agrees with the findings of several studies and supports the hypothesis that underlying mechanisms associated with loss in PW precede diagnosis [3, 4, 9]. The cross-sectional nature of our study does not preclude an alternative hypothesis, namely, that persons at risk for type 1 diabetes have smaller pancreases due to undefined genetic, maternal or environmental factors (reviewed in [1]). Future longitudinal studies in living individuals at high risk for type 1 diabetes and utilising advanced imaging techniques should be used to address issues of pancreatic size in relationship to type 1 diabetes duration and treatment (reviewed in [3]). Here too, studies in type 1 diabetes that would bring clarity to issues beyond those of weight and size alone, including beta cell mass and exocrine inflammation, would also be valuable.

Additional novelty in this report resides in our findings on altered pancreatic regional weights. Specifically, the decline in regional PWs are consistent with primary loss of the pancreatic dorsal lobe, comprised of the major portion of the head and entire body and tail regions, while the ventral lobe (i.e. small portion of head region and most of the uncinate process) remains less affected [10]. In summary, our hope is that the efforts reported herein to normalise PW across a wide age range could stimulate additional studies to further define the clinical and pathogenic significance of reduced PW in type 1 diabetes.

\begin{abstract}
Acknowledgements The authors acknowledge the nPOD staff members and Organ Procurement Organizations, which partner with nPOD to recover organ donors. Additional donor details can be obtained through the JDRF nPOD website (www.jdrfnpod.org). This study used data from the Organ Procurement and Transplantation Network. Donor datasets are available through nPOD DataShare, an online database for collaborative communication organised around the nPOD specimen repository.
\end{abstract}

Funding This work was supported by the JDRF (25-2013-268, 17-2012-3 and 25-2012-516 [MC-T, MAA, AP, JSK]), NIDDK (1DP3DK101120-01 [MC-T, MJH, MAA, DAS]) and 1UL1TR000064 from the National Center for Advancing Translational Science, NIH (JJS).

Duality of interest The authors declare that there is no duality of interest associated with this manuscript.

Contribution statement All authors contributed to the conception and design of the study, data acquisition, analysis and interpretation of the data and manuscript revision, and approved the final version of the paper. MCT and MAA wrote the manuscript and serve as guarantors of the study design, access to data and the decision to submit and publish the manuscript.

\section{References}

1. Battaglia M, Atkinson MA (2015) The streetlight effect in type 1 diabetes. Diabetes 64:1081-1090

2. Campbell-Thompson M, Wasserfall C, Montgomery EL, Atkinson MA, Kaddis JS (2012) Pancreas organ weight in individuals with disease-associated autoantibodies at risk for type 1 diabetes. JAMA 308:2337-2339

3. Williams AJ, Thrower SL, Sequeiros IM et al (2012) Pancreatic volume is reduced in adult patients with recently diagnosed type 1 diabetes. J Clin Endocrinol Metab 97(11):E2109-E2113

4. Gaglia JL, Guimaraes AR, Harisinghani M et al (2011) Noninvasive imaging of pancreatic islet inflammation in type $1 \mathrm{~A}$ diabetes patients. J Clin Invest 121:442-445

5. Henderson JR, Daniel PM, Fraser PA (1981) The pancreas as a single organ: the influence of the endocrine upon the exocrine part of the gland. Gut 22:158-167

6. Foulis AK, Stewart JA (1984) The pancreas in recent-onset type 1 (insulin-dependent) diabetes mellitus: insulin content of islets, insulitis and associated changes in the exocrine acinar tissue. Diabetologia 26:456-461

7. Campbell-Thompson M, Wasserfall C, Kaddis J et al (2012) Network for Pancreatic Organ Donors with Diabetes (nPOD): developing a tissue biobank for type 1 diabetes. Diabetes Metab Res Rev 28:608-617

8. Keenan HA, Sun JK, Levine J et al (2010) Residual insulin production and pancreatic B-cell turnover after 50 years of diabetes: Joslin Medalist Study. Diabetes 59:2846-2853

9. Löhr M, Klöppel G (1987) Residual insulin positivity and pancreatic atrophy in relation to duration of chronic type 1 (insulindependent) diabetes mellitus and microangiopathy. Diabetologia 30:757-762

10. Rahier J, Wallon J, Loozen S, Lefevre A, Gepts W, Haot J (1983) The pancreatic polypeptide cells in the human pancreas: the effects of age and diabetes. J Clin Endocrinol Metab 56:441-444 